\title{
Tendencias de la mortalidad en los departamentos de la región del Pacífico de Colombia (2002-2014)
}

\author{
Mortality trends in the departments of the Pacific region of Colombia (2002-2014)
}

\author{
Tendências da mortalidade nos departamentos da região do Pacífico da \\ Colômbia (2002-2014)
}

Mauricio Pérez-Flórez', Isabel Cristina Ruiz-Buitrago², Jorge Alberto Achcar ${ }^{3}$

1 Estadístico, MSc en Epidemiología, PhD en Salud Pública. Profesor del Departamento de Salud Pública y Epidemiología, Facultad de Ciencias de la Salud, Pontificia Universidad Javeriana, Cali, Colombia. mauricioperez@javerianacali.edu.co. oRciD: https://orcid.org/00000003-0219-9773.

2 Md, especialista en Métodos de Análisis Demográficos. Profesora Universidad Externado de Colombia, Bogotá, Colombia. isacrisrb05@yahoo.com, isabel.ruiz@uexternado.edu.co. oRcID: https://orcid.org/0000-0002-5204-3219.

3 Matemático, MSc en Estadística, PhD en Estadística. Profesor del Departamento de Medicina Social, Facultad de Medicina de Ribeirão Preto, Universidad de São Paulo, Ribeirão Preto, Brasil. achcar@fmrp.usp.br. oRcID: https://orcid.org/0000-0002-9868-9453.

Recibido: 07/10/2018. Aprobado: 22/10/2019. Publicado: 05/02/2019

Pérez-Flórez M, Ruiz-Buitrago IC, Achcar JA. Tendencias de la mortalidad 2002-2014 en los departamentos de la región del Pacífico de Colombia. Rev. Fac. Nal. Salud Pública. 2020;38(1):e335788. doi: https://doi.org/10.17533/udea.rfnsp.v38n1e335788

\section{Resumen}

Objetivo: Describir el perfil y la tendencia de la mortalidad general y específica, durante el periodo 2002-2014, de los departamentos de la región del Pacífico de Colombia: Cauca, Chocó, Nariño y Valle del Cauca. Metodología: Estudio descriptivo-ecológico que usa datos secundarios de mortalidad, nacimientos y proyecciones poblacionales provenientes del Departamento Administrativo Nacional de Estadística. Se calcularon tasas anuales de mortalidad general y específicas (por edad, sexo y para seis grandes grupos de causas de muerte), utilizando la lista corta de clasificación de las causas de mortalidad 6/67 de la Organización Panamericana de la Salud (enfermedades transmisibles, neoplasias/tumores, enfermedades del sistema circulatorio, afecciones originadas en el período perinatal, causas externas y demás causas). Las tendencias fueron evaluadas mediante un modelo de regresión de Poisson con efectos aleatorios. Resultados: Se encontró mayor mortalidad en el sexo masculino (razón hombre / mujer entre $1,26-1,61)$ y en la población mayor a 65 años. Se observó una tendencia descendente en la mortalidad general en los cuatro departamentos, siendo Valle del Cauca el que presentó las mayores tasas de mortalidad general y específica. La principal causa de muerte, y con tendencia ascendente, fueron las enfermedades del sistema circulatorio, seguida de demás causas, causas externas y neoplasias. Conclusiones: La mortalidad general mostró una tendencia descendente, con mayor magnitud en hombres, y riesgo diferencial según sexo a partir de 15-19 años, atribuido al grupo de causas externas. La principal causa de muerte fueron las enfermedades del sistema circulatorio, seguido de todas las demás causas y neoplasias. Las causas externas mostraron una marcada tendencia al descenso.

-Palabras clave: mortalidad, causas de muerte, transición epidemiológica, Colombia. 


\begin{abstract}
Objective: To describe the profile and trend of overall and cause-specific mortality, during the period 2002-2014, of the departments of the Pacific region of Colombia: Cauca, Chocó, Nariño and Valle del Cauca. Methodology: Descriptiveecological study that uses secondary data on mortality, births and population projections from the National Administrative Department of Statistics (DANE). Annual overall and causespecific mortality rates were calculated (by age, sex and for six large groups of causes of death), using the short list of causes of death 6/67 of the Pan American Health Organization (PAHO) (communicable diseases, neoplasms/tumors, diseases of the circulatory system, conditions originating in the perinatal period, external causes and other causes). Trends were evaluated using a Poisson regression model with random effects. Results: Higher mortality was found in males (male/female ratio between 1.26-
\end{abstract}

1.61) and in the population over 65 years of age. A downward trend was observed in overall mortality in the four departments, with Valle del Cauca having the highest overall and causespecific mortality rates. The main cause of death, and with an upward trend, were diseases of the circulatory system, followed by other causes, external causes and neoplasms. Conclusions: Overall mortality showed a downward trend, of greater magnitude in men, and differential risk according to sex from 15-19 years attributed to the group of external causes. The main cause of death was diseases of the circulatory system, followed by all other causes and neoplasms. External causes showed a marked downward trend.

--Keywords: mortality, causes of death, epidemiological transition, Colombia.

\section{Resumo}

Objetivo: Descrever o perfil e a tendência da mortalidade geral e específica, durante o período 2002-2014, dos departamentos da região do Pacífico da Colômbia: Cauca, Chocó, Nariño e Valle del Cauca. Metodologia: Estudo descritivo-ecológico que utiliza dados secundários sobre mortalidade, nascimentos e projeções populacionais do Departamento Administrativo Nacional de Estatística (DANE). Calcularam-se taxas anuais de mortalidade geral e específica (por idade, sexo e para seis grandes grupos de causas de morte), usando a lista curta de classificação de causas de mortalidade 6/67 da Organização Pan-Americana da Saúde (PAHO) (doenças transmissíveis, neoplasias/tumores, doenças do sistema circulatório, condições originadas no período perinatal, causas externas e outras causas). As tendências foram avaliadas através de um modelo de regressão de Poisson com efeitos aleatórios. Resultados: Maior mortalidade foi encontrada no sexo masculino (razão homem / mulher entre 1,26-1,61) e na população acima de 65 anos. Uma tendência descendente na mortalidade geral foi observada nos quatro departamentos, sendo o Valle del Cauca aquele com as maiores taxas de mortalidade geral e específica. As principais causas de morte, e com tendência ascendente, foram as doenças do sistema circulatório, seguidas por outras causas, causas externas e neoplasias. Conclusões: A mortalidade geral apresentou tendência descendente, com maior magnitude nos homens, e risco diferencial segundo o sexo partindo de 15-19 anos, atribuído ao grupo de causas externas. As principais causas de morte foram as doenças do sistema circulatório, seguido por todas as outras causas e neoplasias. As causas externas mostraram uma tendência acentuada ao declínio.

-Palavras-chave: mortalidade, causas de morte, transição epidemiológica, Colômbia.

\section{Introducción}

El análisis de la mortalidad es un tema de interés en diversos campos, que incluyen salud pública, epidemiología, demografía, seguridad social y políticas públicas [1]. Su estudio, según variables como "causa de muerte", "sexo" y "edad", es vital para comprender el perfil epidemiológico de un país o región, y tomar acciones que permitan orientar los servicios y elaborar programas de prevención/intervención para combatir aquellas enfermedades que producen mayor mortalidad. El análisis temporal de la mortalidad también posibilita monitorear cambios en los perfiles epidemiológicos que son propios de cada región, como resultado de condiciones biológicas, sociales, culturales y estructurales en una sociedad. Adicionalmente, la mortalidad es una variable fundamental en el crecimiento de la población y de gran importancia en las proyecciones oficiales de población.

El análisis de las tendencias de la mortalidad y la morbilidad mundial, desde finales del siglo XIX y durante el siglo $\mathrm{xx}$, dio cuenta de la transición epidemiológica que experimentó la población mundial en ese periodo. El concepto de transición epidemiológica se utilizó para denotar cambios en los perfiles poblacionales de salud, enfermedad y muerte, como resultado de otras transformaciones demográficas, económicas 
y sociales. La transición epidemiológica involucró varios fenómenos, donde se destacó el descenso en las tasas de mortalidad y un aumento de la morbilidad; la disminución relativa en la morbimortalidad por enfermedades infecciosas que fueron desplazadas por enfermedades crónicas y degenerativas; la reducción de la mortalidad en la infancia, la cual incrementó en la población más adulta, además de una mixtura con una transición demográfica caracterizada por un crecimiento poblacional, aumento de la esperanza de vida, descenso de las tasas de fertilidad y cambio de la estructura etaria poblacional $[2,3]$.

En América Latina, la transición epidemiológica comenzó hacia los años treinta del siglo xx, presentándose en la región gran heterogeneidad en los perfiles epidemiológicos entre países o en su interior. La carga de morbimortalidad refleja una mixtura de enfermedades de la pre y postransición, lo que permite observar que los países latinoamericanos se hallan en diferentes etapas o están experimentando una nueva y prolongada transición, que podría ser explicada por las desigualdades entre grupos sociales al interior de cada país [4].

Según la Organización Mundial de la Salud (OMS), durante el año 2015 se registraron, en el mundo, 56,4 millones de defunciones, siendo las principales causas de mortalidad, y desde los últimos 15 años, la cardiopatía isquémica $(15,5 \%)$ y el accidente cerebrovascular $(11,1$ \%) [5]. En la región de las Américas se reportaron, ese año, cerca de 6,6 millones de defunciones, con la mayor carga $(80,3 \%)$ para las enfermedades no transmisibles (enfermedades cardiovasculares, cáncer, enfermedades respiratorias crónicas y diabetes), seguidas de las condiciones transmisibles, maternas, perinatales y nutricionales $(10,0 \%)$ y las lesiones $(9,7 \%)$. Estos mismos grupos representaron, respectivamente, el 77,3, 13,1 y $9,6 \%$ de las defunciones del 2000 , cifras que son coherentes con lo postulado por la teoría de la transición epidemiológica en cuanto a un aumento ponderado en la mortalidad por enfermedades no transmisibles y una disminución por las enfermedades infecciosas [1]. Sin embargo, la heterogeneidad en la región se percibe cuando se analizan los perfiles epidemiológicos de países como Colombia, donde tradicionalmente las causas externas (homicidios, accidentes de tránsito y suicidios, principalmente) han tenido un aporte significativo a la carga de mortalidad general [6].

El análisis de la mortalidad para Colombia muestra una tendencia descendente de la tasa bruta de mortalidad entre 2005 y 2014 (de 5,2 a 4,4 defunciones $\times 1.000$ ) [7]. Durante este periodo, la principal causa de muerte fueron las enfermedades del sistema circulatorio $(30,1$ $\%$; tendencia descendente), seguida de neoplasias (18,0 $\%$; tendencia ascendente). Los demás grupos presentaron una tendencia descendente: causas externas $(16,4 \%)$, enfermedades transmisibles $(6,8 \%)$, afecciones del periodo perinatal $(2,4 \%)$ y demás causas $(24,4 \%)$. Sin embargo, los promedios esconden particularidades y las tendencias de mortalidad son propias de cada región, variando incluso en su interior.

Aunque existen diversos estudios que abordan el análisis y las tendencias de la mortalidad, estos se enfocan principalmente en causas o grupos específicos, más que en la mortalidad general [1]. Se encontraron estudios en diferentes contextos que abordaron las tendencias en causas específicas de muerte, como enfermedades cardiovasculares [8], diabetes mellitus [9], causas externas [10,11], homicidios y accidentes de tránsito $[12,13]$ y diferentes tipos de neoplasias $[14,15]$. Estudios sobre tendencias de la mortalidad general se han enfocado en grupos poblacionales específicos; por ejemplo, el estudio sobre las tendencias de mortalidad general en adultos (20-64 años) de Medellín entre 1994-2003 [16]. Sin embargo, se percibe un vacío al no encontrar estudios sobre el nivel, las tendencias y el cambio de los patrones de las causas de mortalidad en los departamentos de la región del Pacífico de Colombia, además de la falta de uso de las fuentes de información disponibles y la necesidad de entregar información para la toma de decisiones, a nivel departamental, para las intervenciones en salud pública.

La región del Pacífico de Colombia es un territorio social y económicamente heterogéneo, con diferentes características sociales, económicas y contextos históricos que modifican sus perfiles de mortalidad; entre aquellas están, por ejemplo, el nivel de desarrollo de Valle del Cauca, que contrasta con el rezago social de Chocó, o el conflicto armado que ha afectado históricamente a poblaciones de Cauca y Nariño.

De esta manera, el objetivo de este artículo es describir el perfil y la tendencia de la mortalidad general y específica, durante el periodo 2002-2014, de los departamentos de la región del Pacífico de Colombia.

\section{Metodología}

\section{Tipo de estudio, periodo y área de estudio}

Estudio descriptivo-ecológico, llevado a cabo en cuatro departamentos de la región del Pacífico colombiano: Cauca, Chocó, Nariño y Valle del Cauca, ubicados en el occidente del país, durante el periodo 2002-2014. Este periodo de estudio fue seleccionado luego de un análisis exploratorio de la información desde el año 2000, que obligó a retirar los resultados de los años 2000 y 2001, por mostrar inconsistencias, y 2014 fue el último año con información disponible al iniciar este estudio.

Estos departamentos abarcan cerca del $11,5 \%$ del territorio nacional y el $18 \%$ de la población del país [17]. Son territorios con diferentes características sociodemográficas y niveles de desarrollo, económico 
y social, como se aprecia en la tabla 1. Una mirada general permite observar que Chocó es el departamento más rezagado. La estructura poblacional de estos departamentos mostró un envejecimiento entre 2002 y 2014, siendo Chocó el departamento que presentó la población más joven $(38,1 \%$ en 2014).

Tabla 1. Características sociodemográficas, económicas y sociales de los cuatro departamentos que comprenden el área de estudio: Cauca, Chocó, Nariño y Valle del Cauca. *

\begin{tabular}{|c|c|c|c|c|}
\hline Variable & Cauca & Chocó & Nariño & Valle del Cauca \\
\hline Número de municipios al año 2005 & 41 & 30 & 64 & 42 \\
\hline Número de habitantes (hab.) al año 2014 & 1.366 .984 & 495.151 & 1.722 .945 & 4.566 .875 \\
\hline Índice de ruralidad (\%) al 2005 & 40,8 & 55,3 & 41,0 & 22,7 \\
\hline Porcentaje de población de etnia negro, mulato, afrocolombiano, en 2005 & 21,6 & 73,6 & 18,1 & 26,9 \\
\hline Población menor de 15 años (\%), en 2002 & 35,1 & 44,2 & 33,9 & 29,8 \\
\hline Población menor de 15 años (\%), en 2014 & 28,8 & 38,1 & 28,7 & 23,8 \\
\hline Población mayor de 60 años (\%), en 2002 & 8,8 & 6,8 & 9,1 & 9,4 \\
\hline Población mayor de 60 años (\%), en 2014 & 10,7 & 6,9 & 10,4 & 12,0 \\
\hline Extensión (km²) & 29.308 & 46.530 & 33.268 & 22.140 \\
\hline Densidad poblacional (hab / km²) & 40,33 & 8,34 & 45,03 & 183,04 \\
\hline Producto interno bruto (PIB) $)^{2}$ per cápita departamental, 2005 (millones de pesos) & 3,594 & 2,585 & 3,080 & 7,585 \\
\hline Índice de desarrollo humano $(\mathrm{IDH}),{ }^{3} 2005$ & 0,73 & 0,67 & 0,72 & 0,79 \\
\hline Índice de desarrollo departamental (IDD), ${ }^{4}$ 2002-2009 & 40,8 & 29,6 & 41,4 & 61,1 \\
\hline Índice de condiciones de vida (ICV), ${ }^{5} 2005$ & 72,0 & 60,5 & 69,3 & 83,0 \\
\hline Necesidades básicas insatisfechas (NBI), ${ }^{6}$ (\%) 2002-2005 & 23,3 & 67,1 & 28,7 & 12,6 \\
\hline Índice de pobreza multidimensional (IPM), 2005 & 70,6 & 85,8 & 68,7 & 38,8 \\
\hline Miseria, ${ }^{8}$ 2002-2005 (\%) & 5,9 & 20,9 & 8,3 & 2,8 \\
\hline Población bajo línea de pobreza (LP), ${ }^{9}$ 2002-2005 (\%) & 61,1 & 78,5 & 64,0 & 56,9 \\
\hline Población bajo línea de indigencia (ㄴ), ${ }^{10} 2002-2005$ (\%) & 27,5 & 48,7 & 23,7 & 19,6 \\
\hline Tasa de desempleo, 2001-2005 & 9,7 & 8,6 & 11,9 & 12,5 \\
\hline Tasa de analfabetismo para población de 15 años y más, 2001-2005 & 8,9 & 22,1 & 9,4 & 4,8 \\
\hline Años promedio de educación para población de 15 años y más, 2001-2005 & 6,5 & 5,6 & 6,4 & 8,1 \\
\hline Cobertura en salud, 2005 (\%) & 58,6 & 55,2 & 63,6 & 71,7 \\
\hline
\end{tabular}

* Las celdas sombreadas en negrilla corresponden al departamento con el peor indicador.

1 Índice de ruralidad = Población rural / Población total.

${ }^{2}$ El PIB expresa el valor monetario de los bienes y servicios producidos en una región y en un periodo determinado.

${ }^{3} \mathrm{El}$ IDH es un indicador que mide el grado de desarrollo humano de un país, departamento o región, con base en tres componentes: esperanza de vida, logro educativo y riqueza.

${ }^{4}$ El IDD fue obtenido como la mediana de los años 2002-2009. Establece el nivel de desarrollo alcanzado por cada departamento, midiendo el comportamiento de los municipios en variables de tipo social y financiero. Menores valores indican menor desarrollo.

${ }^{5}$ El ICV es un indicador multidimensional usado en Colombia, que mide el estándar de vida en una región, combinando doce indicadores relacionados con educación y capital humano; calidad de la vivienda; acceso y calidad de los servicios públicos, y composición del hogar y hacinamiento.

${ }^{6}$ El índice de NBI corresponde al porcentaje de hogares que posee al menos una de las siguientes necesidades básicas insatisfechas: vivienda con materiales inadecuados, con servicios públicos de acueducto y alcantarillado inadecuados, hacinamiento crítico, alta dependencia económica, o cuando uno de sus niños entre 7 y 11 años presenta inasistencia escolar.

${ }^{7}$ El IPM mide el grado de privación de la población en un conjunto de cinco dimensiones, relacionadas con condiciones educativas del hogar; condiciones de la niñez y juventud; trabajo; salud; y acceso a servicios públicos domiciliarios y condiciones de la vivienda. Una persona es multidimensionalmente pobre si no supera la tercera parte de las dimensiones seleccionadas (5/15) o no supera el 33,3\% según la ponderación asignada.

${ }^{8}$ El índice de miseria corresponde al porcentaje de hogares que presenta al menos dos necesidades básicas insatisfechas, mencionadas en el índice de NBI.

${ }^{9}$ La LP estima el porcentaje de hogares cuyos ingresos es inferior a un valor dado, lo que les impide adquirir una canasta básica alimentaria.

${ }^{10}$ La LI estima el porcentaje de hogares cuyos ingresos son insuficientes para cubrir los requerimientos nutricionales mínimos de cada miembro del hogar.

Fuente: Datos demográficos de las proyecciones de población departamentales por área 2005-2020 del Departamento Nacional de Estadística (DANE) [17]; PIB de las Cuentas departamentales del DANE; extensión territorial de los departamentos de la Sociedad Geográfica de Colombia [18]; e indicadores sociales departamentales del Departamento Nacional de Planeación [19]. 


\section{Variables y fuente de información}

Los datos de mortalidad provienen de las "Estadísticas Vitales" disponibles en el Departamento Nacional de Estadística (DANE) [20], entidad oficial que reúne la información de los certificados de defunción del país.

De la información disponible se usaron variables sociodemográficas relacionadas con el fallecido (sexo, edad y municipio de residencia) y con la defunción (fecha, municipio de ocurrencia y causa básica de muerte, codificada con los cuatro dígitos de la "Clasificación Internacional de Enfermedades versión 10" — CIE-10 ). También se obtuvo del DANE el número de nacidos vivos (NV) y las proyecciones poblacionales a partir del último censo (año 2005) [17].

\section{Procedimientos}

El análisis de la mortalidad para cada departamento se realizó a partir de: 1) la tasa bruta de mortalidad, estandarizada por edad, por medio del método de ajuste directo, con población estándar Colombia del año 2005; y 2) la tasa de mortalidad específica (total y según sexo), para seis grandes grupos: 1) enfermedades transmisibles; 2) neoplasias/tumores; 3) enfermedades del sistema circulatorio; 4) afecciones originadas en el periodo perinatal (en adelante, "perinatal"); 5) causas externas, y 6) todas las demás causas. La creación de estos grupos se basó en la lista de mortalidad 6/67 de la Organización Panamericana de la Salud (ops) [21], que agrupa todas las muertes en 6 grandes grupos y 67 subgrupos más detallados con base en el CIE-10.

Para asignar cada defunción a su respectivo municipio de análisis, se usó la variable "lugar de residencia", excepto para las causas externas, donde se utilizó "lugar ocurrencia".
Durante el periodo 2002-2014 fueron reportadas 462.482 defunciones en los departamentos de la región del Pacífico, de las cuales se eliminó el 0,66 \% (3.058 / 462.482) de los registros, por tener información demográfica faltante del sexo $(n=45)$, edad $(n=1.790)$ o municipio de residencia/ocurrencia $(n=1.223)$. Del total, se analizaron 459.424 defunciones en este estudio.

Al ser muy baja la información faltante en las variables requeridas, no fue necesario efectuar un proceso de imputación de datos ni se consideró relevante la redistribución de estas defunciones. Tampoco se hizo ningún proceso de ajuste por subregistro a la base de datos de mortalidad oficial del DANE.

\section{Calidad de la información}

Como es recomendado en los estudios que utilizan fuentes de datos secundarios, se hizo una evaluación aproximada de la calidad de los datos de mortalidad, con el uso de la información del último año (2014). Se usó el software ANACONDA ${ }^{\circledR}$ Versión 3.1.0, para evaluar la calidad de la información y asegurar que se producen patrones coherentes de mortalidad. anaconda ${ }^{\circledR}$ confronta los datos de interés con las estimaciones más recientes del país (o una región vecina), realizadas para el "Estudio de carga global de enfermedad" [22]. Al final se calcula un índice de calidad de los datos (global y por componente), llamado "Índice de desempeño de las estadísticas vitales" en una escala de 0 a 100 [23].

Los resultados de este análisis (véase tabla 2) permitieron concluir que la calidad de la información de mortalidad se considera alta para Valle del Cauca y media para Cauca, Chocó y Nariño.

Tabla 2. Índice de desempeño de las estadísticas vitales (global y por componente) para los datos de mortalidad del año 2014 de los departamentos del área de estudio.

\begin{tabular}{lcccc}
\hline \multicolumn{1}{c}{ Componente } & Cauca & Chocó & Nariño & Valle del Cauca \\
\hline Calidad de la información sobre edad y sexo (\%) & 100,0 & 99,3 & 100,0 & 100,0 \\
Calidad de la información sobre la causa de muerte (\%) & 88,2 & 84,1 & 88,2 & 89,5 \\
Causa de muerte biológicamente plausible (\%) & 100,0 & 100,0 & 100,0 & 100,0 \\
Nivel de detalle de la causa específica disponible (\%) & 88,0 & 80,9 & 88,8 & 90,9 \\
Integridad de la declaración de defunción (\%) & 88,6 & 95,2 & 84,0 & 91,9 \\
Índice global (\%) & 68,8 & 64,3 & 65,8 & 74,8 \\
\hline
\end{tabular}

Fuente: Resultados obtenidos del software ANACONDA ${ }^{\circledR}$ Versión 3.1.0. 
La lista de mortalidad 6/67 de la ops [21] también define un grupo residual de "síntomas, signos y afecciones mal definidas", que frecuentemente es usado como un indicador de calidad. Al usar este indicador, se encontró que durante el periodo 2002-2014, Valle del Cauca reportó la menor proporción de defunciones mal definidas (1,0\%), seguido de Nariño (3,0\%), Cauca $(4,0$ \%) y Chocó $(4,4 \%)$.

\section{Análisis de la información}

Comprendió un análisis descriptivo de la mortalidad, cálculo de tasas de mortalidad general y específica (según sexo, edad y causa) y graficas de tendencias. Las tasas de mortalidad por edad (en grupos quinquenales) fueron presentadas gráficamente por medio de curvas de mortalidad para tres periodos (2002-2005, 2006-2010 y 2011-2014), y en escala logaritmo, para apreciar mejor el patrón por grupo etario. La sobremortalidad masculina fue reportada mediante la razón entre la tasa de mortalidad masculina y la femenina: razón (hombre / mujer).

La tasa bruta de mortalidad se obtuvo por medio del cociente entre el número total de defunciones y el total poblacional $(\times 1.000)$. La tasa de mortalidad por causa específica " $X$ " dividió el número de muertes por causa específica "X" entre el total poblacional (× 100.000), excepto para perinatal, donde el denominador fueron NV $(\times 1.000)$. Todas las tasas de mortalidad específicas reportadas en los "Resultados" y la "Discusión" se multiplicaron por 100.000 para facilitar su interpretación.

Los seis grandes grupos de causas de muerte fueron desagregados en subgrupos de la lista de mortalidad 6/67 de ops (causas de tres dígitos), según sexo y para los tres periodos definidos. Estos subgrupos se seleccionaron de tal forma que ellos acumularan el $95 \%$ de las defunciones totales del grupo.

Para evaluar la tendencia anual de la mortalidad (por departamento, sexo y causa), se usaron modelos de regresión Poisson con efecto aleatorio $(W)$, bajo un esquema bayesiano. Para la modelación se definió, como variable dependiente, la "tasa anual de mortalidad" en cada municipio $\left(\theta_{i}\right)(i=1,2, \ldots, n)$ y la variable independiente fue $A N O_{i}$, representando el año $i$ de ocurrencia de la defunción $\left(A \tilde{N} O_{i}=1,2, \ldots, 13\right.$, que representa respectivamente de 2002 a 2014). El tamaño de muestra $n$ en estos análisis fue determinado por el número de municipios al 2005 de cada departamento (véase tabla 1).
La tasa anual de mortalidad $\left(\theta_{i}\right)$ se obtuvo al dividir el número de muertes del municipio $i\left(Y_{i}\right)$, entre el número de habitantes del municipio $\left(N_{i}\right)$, es decir, $\theta_{i}=Y_{i} / N_{i}$. Se asumió que Yi tiene una distribución de Poisson con parámetro $\lambda_{i}$ :

$$
\begin{gathered}
Y_{i} \sim \text { Poisson }\left(\lambda_{i}\right) \\
\log \left(\lambda_{i}\right)=\log N_{i}+\beta_{0}+\left(\beta_{1} \times A \tilde{N} O_{i}\right)+W_{i}, \text { donde } i=1, \ldots, n .
\end{gathered}
$$

En este modelo, el parámetro de regresión $\beta_{1}$ evalúa la tendencia anual ascendente $(\uparrow)$, descendente $(\downarrow)$ o estable de la mortalidad, el cual se interpreta como el cambio en el número esperado de muertes (escala logaritmo) por cada año que transcurre. Solo se reportó el parámetro $\beta 1 \mathrm{y}$ su intervalo de credibilidad (equivalente a un intervalo de confianza bajo un esquema clásico) del $95 \%\left(\mathrm{IC}_{\text {Cred }} 95 \%\right)$ cuando este fue estadísticamente significativo, es decir, cuando el $\mathrm{IC}_{\text {Cred }} 95 \%$ no contiene el cero. El análisis fue realizado en el software Openbugs ${ }^{\circledR}$ Versión 3.2.3 [24], asumiendo distribuciones a priori, aproximadamente no informativas en todos los casos, y una distribución normal para el efecto aleatorio $(W)[25]$.

\section{Resultados}

\section{Caracterización y tendencias de la mortalidad general}

Valle del Cauca aportó el 66,3 \% (304.503 / 459.424) de las defunciones totales del periodo de estudio, seguido de Nariño, con 16,1 \% (73.976 / 459.424); Cauca, 14,3 \% (65.488 / 459.424), y Chocó, 3,4 \% (15.457 / 459.424).

En la región del Pacífico, el 58,4 \% (268.143 / 459.424) de las defunciones ocurrieron en hombres, $\mathrm{y}$ el 41,6 \% (191.281 / 459.424) en mujeres; el análisis por departamentos también mostró mayor mortalidad masculina. Durante el periodo de estudio se observó una reducción en la proporción de muertes masculinas, siendo para los periodos 2002-2005, 2006-2010 y 20112014 , respectivamente, $59,3,58,2$ y $57,7 \%$.

Se encontró que, durante el periodo de estudio, Valle del Cauca presentó las mayores tasas de mortalidad general (véase figura 1a). Las tasas estandarizadas de mortalidad mostraron una tendencia descendente en los cuatro departamentos durante el periodo 2002-2014, siendo el descenso más importante en Valle del Cauca (véase figura 1a). Este descenso también se observó en las tasas específicas de mortalidad masculina de Valle del Cauca (véase figura 1b). 

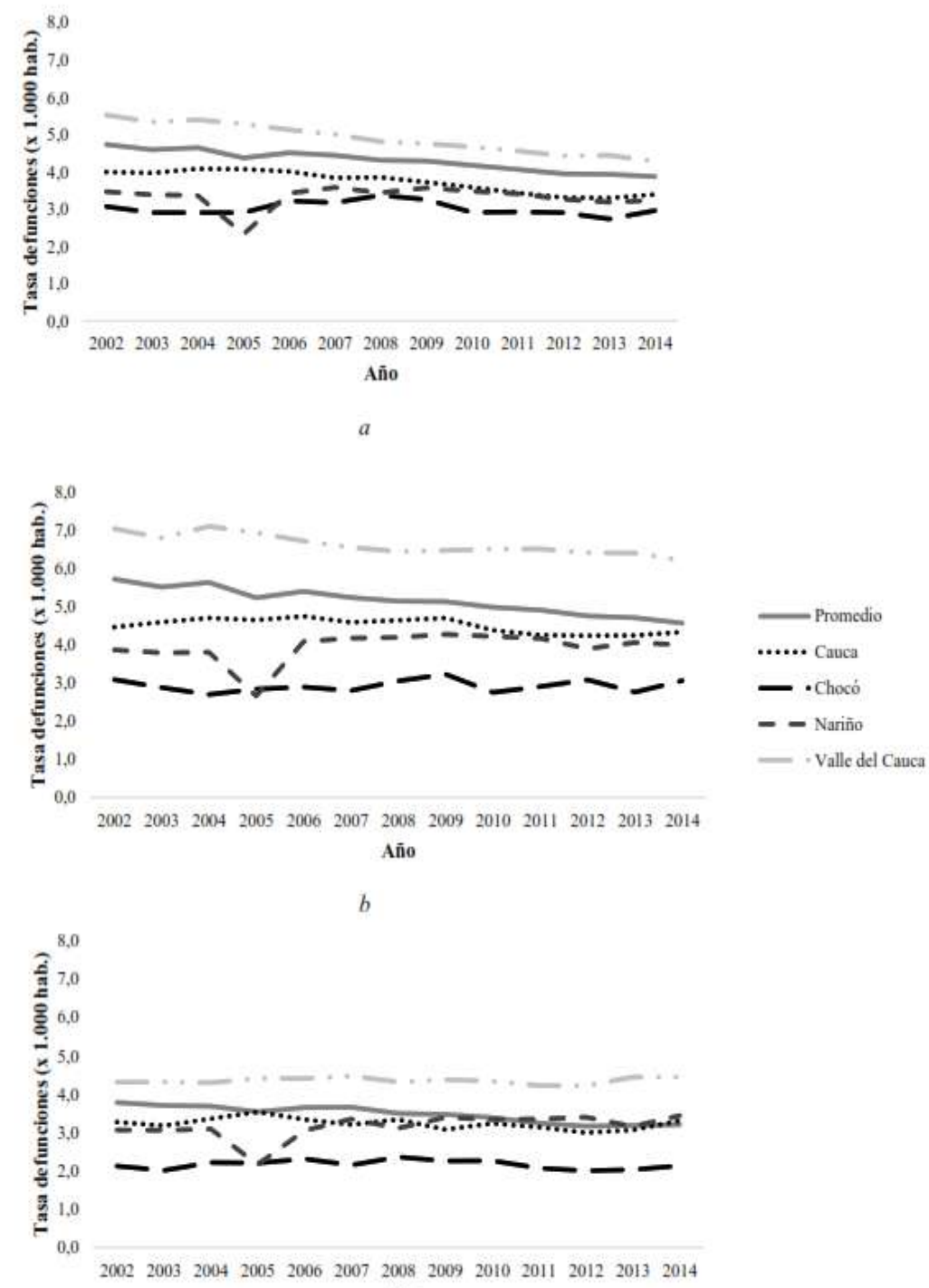

Año

$c$

Figura 1. Tendencias de la mortalidad por departamentos de la región del Pacífico durante los años 2002 a 2014 . a. Tasa estandarizada de mortalidad por departamentos y promedio; b. tasa específica de mortalidad en hombres; c. tasa específica de mortalidad en mujeres.

Fuente: Elaboración propia a partir de los datos de mortalidad del DANE y las proyecciones de población departamentales por área 2005-2020 del DANE [17].

Las razones de mortalidad (hombre / mujer) fueron homogéneas a lo largo del periodo de estudio, con valores promedio de 1,26 en Nariño; 1,33 en Chocó; 1,40 en Cauca y 1,61 en Valle del Cauca (véase figuras 1 b y 1c).

En la región del Pacífico, el análisis de la mortalidad por grupos etarios mostró que el 52,6 \% de las muertes ocurrieron en personas mayores de 65 años, seguido del grupo de 15 a 44 años $(22,1 \%)$ y de 45 a 64 años (18,8\%). A nivel departamental, el comportamiento fue similar, aunque en Chocó el grupo de 65 o más años representó solo el 37,8 \% de las muertes, mientras que el
$16,0 \%$ fueron en niños menores de un año, y el 2,0 \%, en niños de 1 a 4 años.

Las tasas específicas de mortalidad por grupo etario mostraron que, de 2002 a 2014, Chocó y Cauca registraron las mayores tasas en menores de 1 año (14,03 y $13,08 \times 1.000$, respectivamente), en comparación con Nariño y Valle del Cauca $(10,04$ y 10,74 × 1.000, respectivamente). Chocó también tuvo las mayores tasas de mortalidad en los grupos de 1 a 4 y de 5 a 9 años, mientras que a partir del grupo de 10 a 14 años, Valle del Cauca presentó las mayores tasas de mortalidad. 
En general, las curvas de mortalidad (véanse figuras $2 \mathrm{a}$ a $2 \mathrm{~h}$ ) muestran un alto riesgo de morir en los primeros años de vida, alcanzando su mínimo en el grupo de 5 a 9 años en Cauca, Nariño y Valle del Cauca, y en el grupo de 10 a 14 años en Chocó. A partir del grupo de 15 a 19 años, el riesgo de muerte aumenta diferencialmente
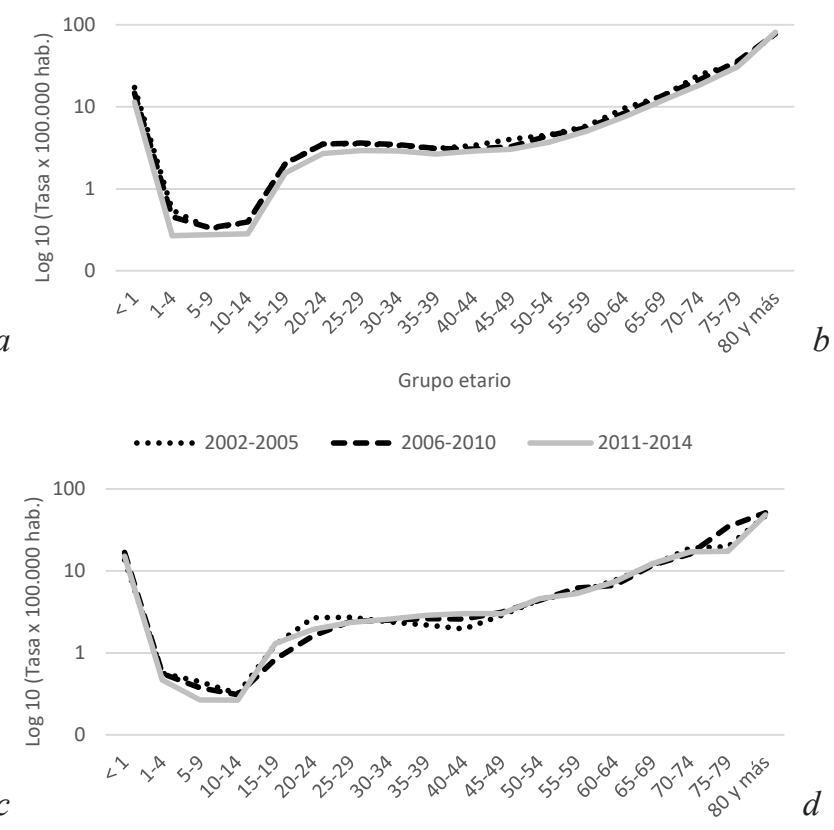

Grupo etario

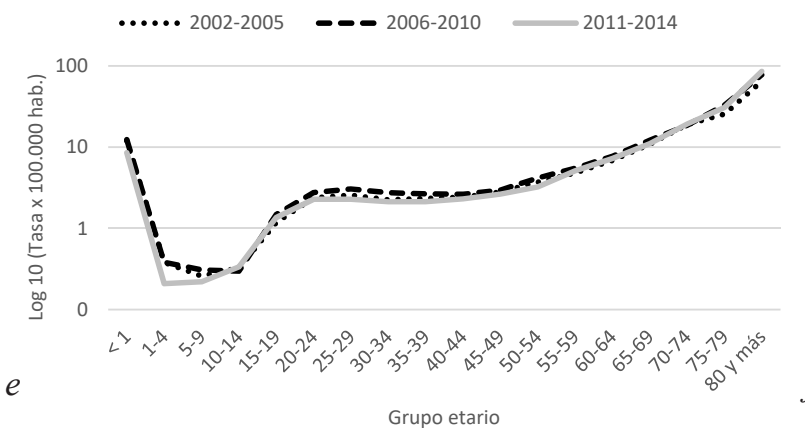

Grupo etario

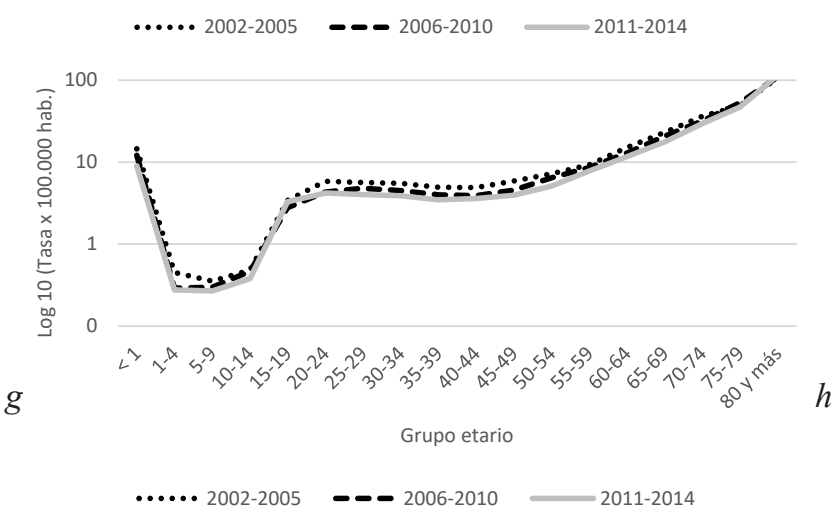

entre hombres y mujeres, observando la mayor sobremortalidad masculina en el grupo de 20 a 29 años en los cuatro departamentos. También se observó una tendencia descendente de la mortalidad en todos los departamentos y grupos etarios durante los tres periodos definidos.

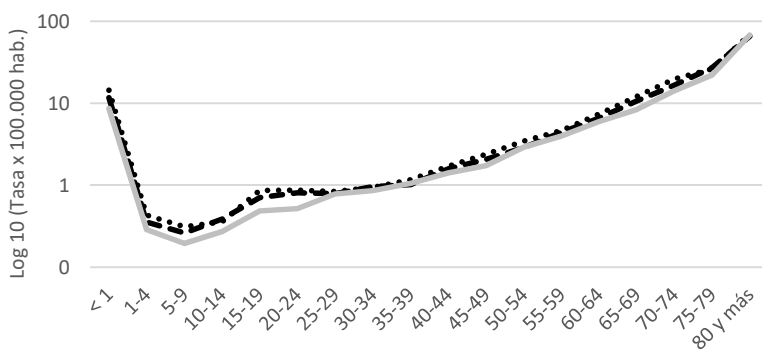

Grupo etario

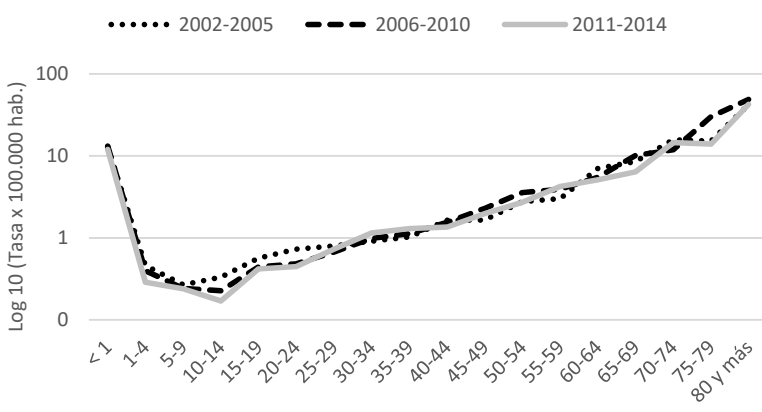

Grupo etario

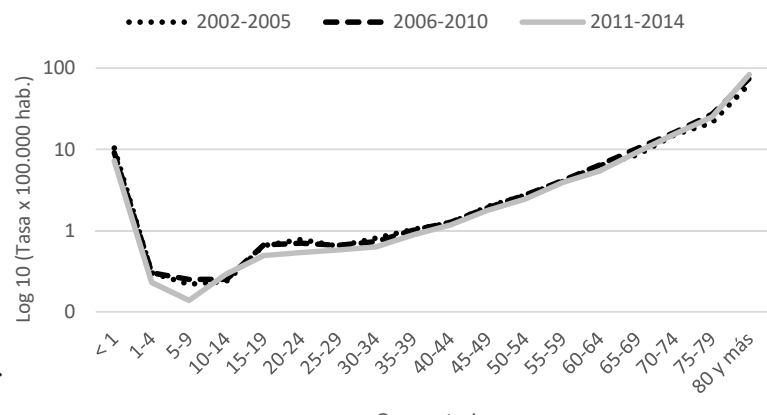

Grupo etario

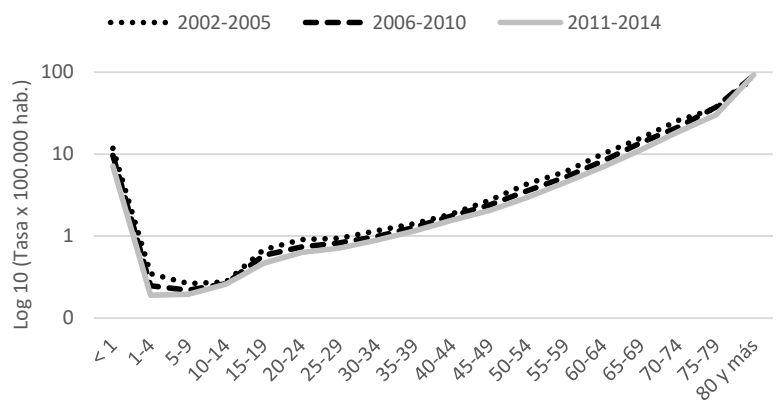

Grupo etario

....2 2002-2005 2011-2014

Figura 2. Curvas de mortalidad según el sexo en los departamentos de la región del Pacífico de Colombia, periodos 2002-2005, 2006-2010 y 2011-2014. a. Cauca-hombres; b. Cauca-mujeres; c. Chocó-hombres; d. Chocó-mujeres; e. Nariño-hombres; f. Nariño-mujeres; g. Valle del Cauca-hombres; h. Valle del Cauca-mujeres. 


\section{Mortalidad proporcional por grandes grupos de causas}

En el período de análisis 2002-2014, en la región del Pacífico, el orden de presentación de las causas se ha modificado (véase tabla 3). Se encontró que, desde el 2002, las enfermedades del sistema circulatorio se han mantenido como la principal causa de muerte, aumentando de $27,2 \%$ en 2002 a $29,6 \%$ en 2014 $(+2,4 \%)$, y desde 2005 , todas las demás causas se han mantenido como la segunda causa de muerte $(+4,0 \%)$, mientras que las neoplasias finalizaron en el tercer lugar $(+3,9 \%)$. Las causas externas han descendido, pasando del segundo lugar al cuarto, con una disminución sostenida de la proporción durante el período $(-7,9 \%)$. Las enfermedades transmisibles $(-1,2 \%)$ y las afecciones del periodo perinatal $(-1,2 \%)$ se mantuvieron como la quinta y sexta causa de muerte durante todo el periodo de estudio.

Tabla 3. Tasas de mortalidad ( $\times 100.000)$ por subgrupos de la lista de mortalidad $6 / 67$ de la ops y sexo, para los periodos 2002-2005, 2006-2010 y 2011-2014 en la región del Pacífico de Colombia

Orden de importancia de las causas de muerte (mortalidad proporcional)

$$
\begin{array}{|l|l|l|l|l|l|}
1 & 2 & 3 & 4 & 5 & 6 \\
\hline
\end{array}
$$

\begin{tabular}{lccccccccccccc}
\hline \multicolumn{1}{c}{ Grupo } & $\mathbf{2 0 0 2}$ & $\mathbf{2 0 0 3}$ & $\mathbf{2 0 0 4}$ & $\mathbf{2 0 0 5}$ & $\mathbf{2 0 0 6}$ & $\mathbf{2 0 0 7}$ & $\mathbf{2 0 0 8}$ & $\mathbf{2 0 0 9}$ & $\mathbf{2 0 1 0}$ & $\mathbf{2 0 1 1}$ & $\mathbf{2 0 1 2}$ & $\mathbf{2 0 1 3}$ & $\mathbf{2 0 1 4}$ \\
\hline Enfermedades del sistema circulatorio & 27,2 & 26,8 & 27,4 & 28,5 & 28,0 & 27,8 & 27,9 & 28,1 & 27,9 & 27,9 & 28,3 & 28,8 & 29,6 \\
\hline Todas las demás causas & 20,6 & 22,5 & 20,8 & 21,9 & 22,9 & 22,7 & 24,4 & 22,7 & 23,4 & 23,1 & 23,4 & 23,6 & 24,6 \\
\hline Neoplasias & 15,6 & 16,1 & 16,1 & 16,5 & 16,8 & 17,1 & 17,0 & 17,5 & 18,0 & 17,5 & 18,3 & 19,0 & 19,5 \\
\hline Causas externas & 24,9 & 22,1 & 23,7 & 20,7 & 20,4 & 20,5 & 19,8 & 21,0 & 20,7 & 20,6 & 19,8 & 18,8 & 16,9 \\
\hline Enfermedades transmisibles & 7,1 & 8,1 & 7,6 & 8,3 & 8,1 & 8,0 & 6,6 & 6,5 & 6,4 & 6,3 & 6,8 & 6,7 & 6,0 \\
\hline $\begin{array}{l}\text { Ciertas afecciones originadas en el } \\
\text { periodo perinatal }\end{array}$ & 2,8 & 2,5 & 2,7 & 2,5 & 2,4 & 2,3 & 2,2 & 2,1 & 1,6 & 1,7 & 1,8 & 1,5 & 1,5 \\
\hline
\end{tabular}

En general, la mortalidad proporcional, a nivel departamental, se comporta de manera similar a la mortalidad de la región (véase tabla 3). Se resalta que en Chocó se observó la mayor mortalidad proporcional debida a enfermedades transmisibles (promedio $10,1 \%$ ) y perinatal (promedio 7,3\%), y el descenso diferencial de la mortalidad por causas externas entre hombres $(-10,5 \%)$ y mujeres $(-2,4 \%)$.

\section{Mortalidad específica por grandes grupos de causas}

La figura 3 muestra las tendencias de las tasas de mortalidad por causas específicas (total y según sexo) durante el periodo 2002-2014, y la tabla 4 contiene las tasas específicas de mortalidad de los subgrupos dentro de los seis grandes grupos de causas para los tres periodos definidos. En general, las mayores tasas de mortalidad para todos los grupos se observaron en Valle del Cauca (excepto para perinatal), y las menores se observaron en Chocó (excepto para enfermedades transmisibles). 


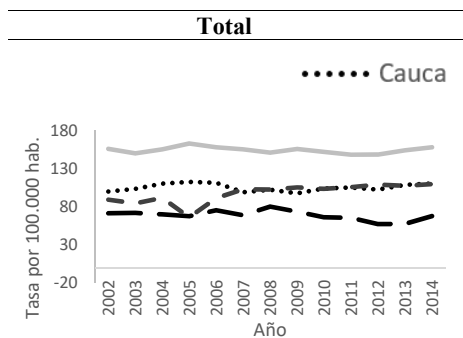

个Nariño: $\beta_{1}=0,0289(0,017 ; 0,042)^{*}$

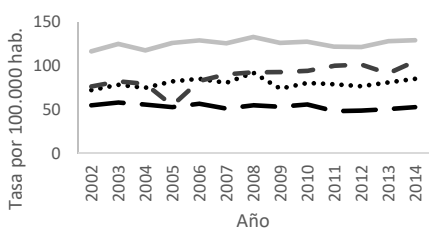

$\uparrow$ Nariño: $\beta_{1}=0,0263(0,016 ; 0,037)$ $\uparrow$ Valle del Cauca: $\beta_{1}=0,0073(0,001 ; 0,014)$

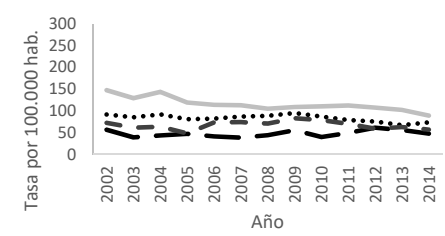

Valle del Cauca: $\beta_{1}=-0,036(-0,043 ;-0,028)$

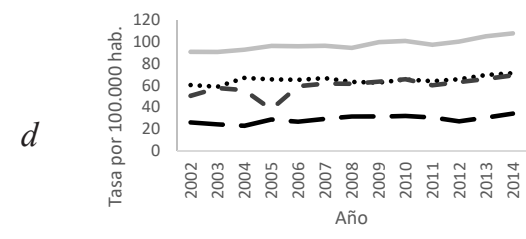

个Nariño: $\beta_{1}=0,0195(0,007 ; 0,032)$

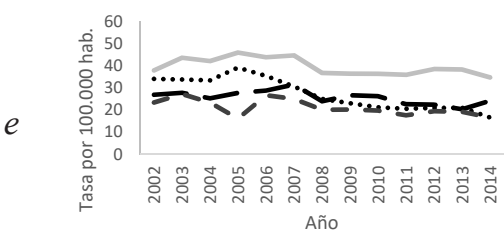

$\downarrow_{\text {Cauca: }} \beta_{1}=-0,0474(-0,059 ;-0,035)$ $\downarrow_{\text {Nariño: }} \beta_{1}=-0,0195(-0,031 ;-0,009)$ $\downarrow$ Valle del Cauca: $\beta_{1}=-0,0124(-0,02 ;-0,004)$

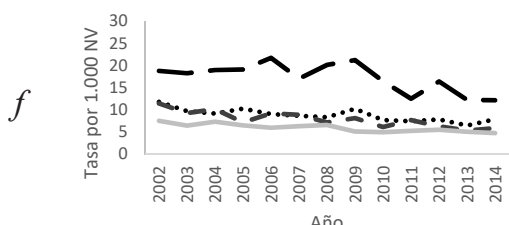

Hombres

Mujeres
Chocó
- Nariño
- Valle del Cauca

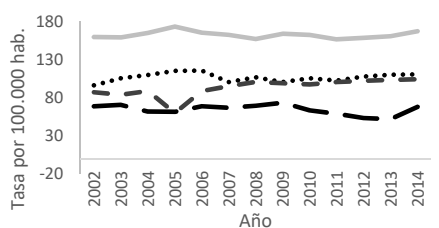

$\uparrow$ Nariño: $\beta_{1}=0,0261(0,014 ; 0,038)$

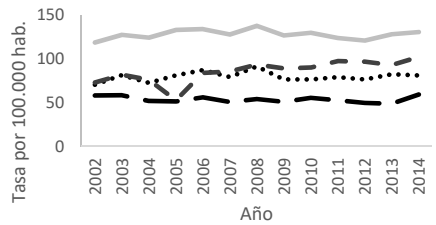

$\uparrow$ Nariño: $\beta_{1}=0,0252(0,014 ; 0,037)$

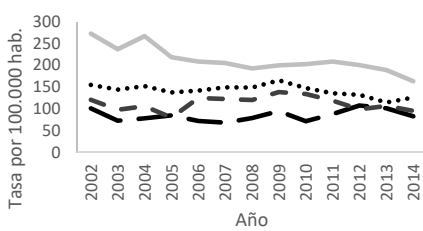

$\downarrow$ Valle del Cauca: $\beta_{1}=-0,036(-0,043 ;-0,028)$

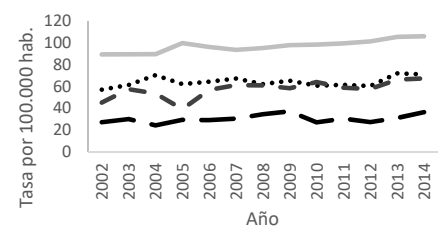

$\uparrow$ Nariño: $\beta_{1}=0,0213(0,009 ; 0,032)$

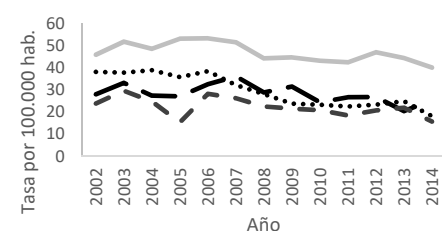

Año

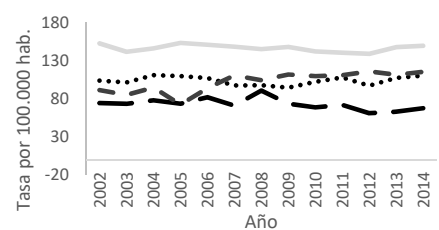

个Nariño: $\beta_{1}=0,0284(0,016 ; 0,041)$

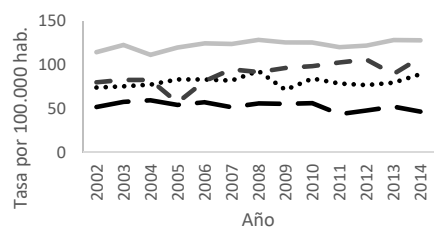

$\uparrow$ Nariño: $\beta_{1}=0,0237(0,013 ; 0,034)$

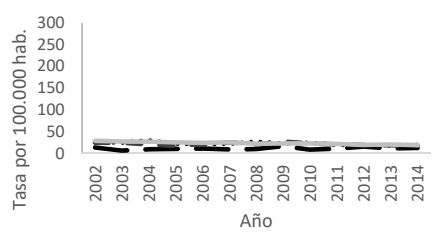

$\downarrow_{\text {Cauca: }} \beta_{1}=-0,0165(-0,03 ;-0,002)$ $\downarrow$ Valle del Cauca: $\beta_{1}=-0,0237(-0,034 ;-0,013)$

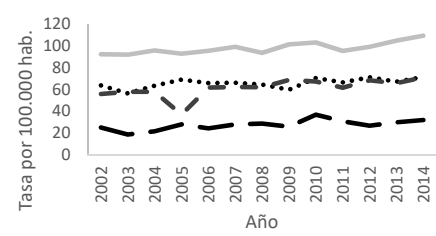

$\uparrow$ Nariño: $\beta_{1}=0,0139(0,002 ; 0,026)$

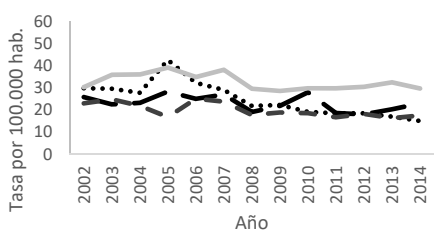

Año

\section{$\downarrow$ Cauca: $\beta_{1}=-0,0441(-0,058 ;-0,031)$} $\downarrow$ Nariño: $\beta_{1}=-0,0162(-0,029 ;-0,003)$ $\downarrow$ Valle del Cauca: $\beta_{1}=-0,0125(-0,022 ;-0,003)$ $\downarrow$ Valle del Cauca: $\beta_{1}=-0,0113(-0,02 ;-0,001)$
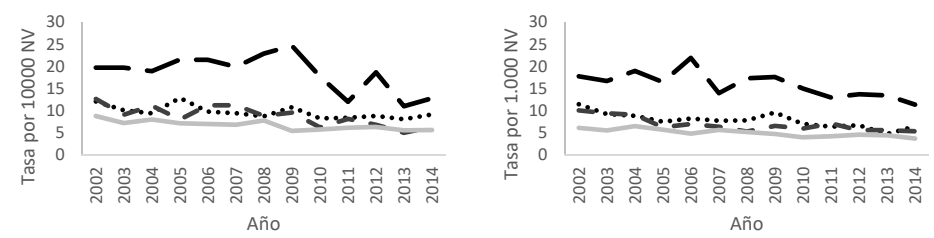

Figura 3. Tasa de mortalidad específica por causa de muerte en todos los departamentos de la región del Pacífico colombiano, durante los años 2002 al 2014, total y según sexo: a. enfermedades del sistema circulatorio; b. todas las demás enfermedades; c. causas externas; d. neoplasias (tumores); e. enfermedades transmisibles; f. ciertas afecciones originadas en el período perinatal.

NV: Nacidos vivos.

* El valor entre paréntesis representa el intervalo de credibilidad del $95 \%$

Fuente: Elaboración propia, a partir de la información de "Estadisticas Vitales" de mortalidad del DANE [20] y las proyecciones de población departamentales por área 2005-2020 del DANE [17]. 
Tabla 4. Tasas de mortalidad ( $\times 100.000$ ) por subgrupos de la lista de mortalidad $6 / 67$ de ops/oms y sexo para los periodos 2002-2005, 2006-2010 y 2011-2014 en la región del Pacífico de Colombia.

\begin{tabular}{|c|c|c|c|c|c|c|}
\hline \multirow{2}{*}{$\begin{array}{c}\text { Subgrupos } \\
\text { (Causa de muerte con } 3 \text { dígitos) }\end{array}$} & \multicolumn{3}{|c|}{ Hombres } & \multicolumn{3}{|c|}{ Mujeres } \\
\hline & $2002-2005$ & $2006-2010$ & 2011-2014 & $2002-2005$ & 2006-2010 & 2011-2014 \\
\hline \multicolumn{7}{|c|}{ Enfermedades del sistema circulatorio (Tasas $\times 100.000$ ) } \\
\hline Enfermedades isquémicas del corazón & 63,03 & 66,88 & 68,56 & 49,39 & 52,01 & 54,40 \\
\hline Enfermedades cerebrovasculares & 30,97 & 31,57 & 30,98 & 35,72 & 37,75 & 36,55 \\
\hline Enfermedades hipertensivas & 13,66 & 13,04 & 15,05 & 15,77 & 14,47 & 16,64 \\
\hline $\begin{array}{l}\text { Enfermedad cardiopulmonar, enfermedades } \\
\text { de la circulación pulmonar y otras formas de } \\
\text { enfermedad del corazón }\end{array}$ & 9,39 & 8,92 & 8,82 & 10,48 & 10,26 & 10,04 \\
\hline Insuficiencia cardíaca & 6,53 & 6,10 & 4,72 & 6,59 & 6,78 & 5,07 \\
\hline Demás del grupo & 7,22 & 6,50 & 5,10 & 6,48 & 5,57 & 4,42 \\
\hline \multicolumn{7}{|c|}{ Todas las demás enfermedades (Tasas $\times 100.000$ ) } \\
\hline $\begin{array}{l}\text { Enfermedades crónicas de las vías } \\
\text { respiratorias inferiores }\end{array}$ & 22,89 & 24,49 & 23,55 & 17,02 & 18,33 & 18,89 \\
\hline Diabetes mellitus & 15,87 & 14,75 & 13,99 & 19,90 & 18,89 & 18,11 \\
\hline Resto de enfermedades del sistema digestivo & 13,93 & 14,85 & 14,02 & 13,34 & 14,82 & 14,34 \\
\hline Resto de las enfermedades & 7,28 & 9,70 & 8,35 & 9,77 & 15,11 & 13,78 \\
\hline Enfermedades del sistema urinario & 9,25 & 11,39 & 11,84 & 8,43 & 10,63 & 10,86 \\
\hline $\begin{array}{l}\text { Enfermedades del sistema nervioso, excepto } \\
\text { meningitis }\end{array}$ & 5,04 & 6,04 & 6,67 & 3,96 & 4,71 & 5,65 \\
\hline $\begin{array}{l}\text { Malformaciones congénitas, deformidades y } \\
\text { anomalías cromosómicas }\end{array}$ & 6,27 & 6,34 & 5,19 & 5,12 & 4,96 & 4,17 \\
\hline Otras enfermedades respiratorias & 4,86 & 5,68 & 6,58 & 4,33 & 4,80 & 5,69 \\
\hline $\begin{array}{l}\text { Deficiencias nutricionales y anemias } \\
\text { nutricionales }\end{array}$ & 4,95 & 4,48 & 4,49 & 5,40 & 4,58 & 4,83 \\
\hline $\begin{array}{l}\text { Cirrosis y ciertas otras enfermedades crónicas } \\
\text { del hígado }\end{array}$ & 5,58 & 5,33 & 5,62 & 3,84 & 3,93 & 4,15 \\
\hline $\begin{array}{l}\text { Apendicitis, hernia de la cavidad abdominal y } \\
\text { obstrucción intestinal }\end{array}$ & 2,37 & 3,09 & 3,10 & 2,69 & 3,02 & 3,09 \\
\hline Demás del grupo & 2,72 & 2,39 & 3,65 & 4,22 & 3,50 & 4,34 \\
\hline \multicolumn{7}{|l|}{ Causas externas (Tasas $\times 100.000)$} \\
\hline Agresiones (homicidios), inclusive secuelas & 134,31 & 115,16 & 107,32 & 10,29 & 9,63 & 8,67 \\
\hline $\begin{array}{l}\text { Accidentes de transporte terrestre, inclusive } \\
\text { secuelas }\end{array}$ & 26,67 & 26,01 & 23,69 & 6,88 & 6,34 & 5,48 \\
\hline $\begin{array}{l}\text { Lesiones autoinfligidas intencionalmente } \\
\text { (suicidios), inclusive secuelas }\end{array}$ & 8,80 & 8,87 & 7,33 & 3,05 & 2,66 & 1,53 \\
\hline Otros accidentes, inclusive secuelas & 4,69 & 4,93 & 4,37 & 1,21 & 1,14 & 0,82 \\
\hline Caídas & 3,88 & 4,04 & 3,44 & 1,09 & 1,00 & 1,08 \\
\hline $\begin{array}{l}\text { Eventos de intención no determinada, } \\
\text { inclusive secuelas }\end{array}$ & 3,84 & 3,73 & 3,49 & 0,97 & 0,66 & 0,61 \\
\hline Demás del grupo & 7,36 & 6,79 & 5,52 & 1,35 & 1,50 & 1,19 \\
\hline \multicolumn{7}{|l|}{ Neoplasias (Tumores) (Tasas $\times 100.000)$} \\
\hline Tumor maligno del estómago & 15,81 & 15,47 & 14,77 & 10,81 & 10,38 & 9,48 \\
\hline Otros tumores malignos & 11,17 & 10,59 & 11,33 & 11,20 & 11,22 & 11,06 \\
\hline $\begin{array}{l}\text { Tumor maligno de la tráquea, los bronquios y } \\
\text { el pulmón }\end{array}$ & 10,17 & 10,12 & 9,51 & 6,17 & 6,58 & 6,52 \\
\hline $\begin{array}{l}\text { Tumor maligno del tejido linfático, de los } \\
\text { órganos hematopoyéticos y de tejidos afines }\end{array}$ & 6,74 & 7,57 & 8,01 & 5,49 & 6,30 & 6,79 \\
\hline Tumor maligno de la próstata & 11,37 & 12,12 & 12,63 & $\mathrm{~N} / \mathrm{A}$ & N/A & N/A \\
\hline Tumor maligno de hígado y vías biliares & 4,47 & 5,23 & 5,05 & 6,79 & 7,08 & 6,73 \\
\hline Tumor maligno de la mama de la mujer & N/A & $\mathrm{N} / \mathrm{A}$ & $\mathrm{N} / \mathrm{A}$ & 8,87 & 10,12 & 10,99 \\
\hline Tumor maligno del útero & $\mathrm{N} / \mathrm{A}$ & $\mathrm{N} / \mathrm{A}$ & $N / A$ & 10,87 & 9,84 & 9,47 \\
\hline Tumor maligno del colon & 2,93 & 3,65 & 4,63 & 3,88 & 4,88 & 5,22 \\
\hline
\end{tabular}




\begin{tabular}{|c|c|c|c|c|c|c|}
\hline \multirow{2}{*}{$\begin{array}{c}\text { Subgrupos } \\
\text { (Causa de muerte con } 3 \text { dígitos) }\end{array}$} & \multicolumn{3}{|c|}{ Hombres } & \multicolumn{3}{|c|}{ Mujeres } \\
\hline & 2002-2005 & $2006-2010$ & 2011-2014 & $2002-2005$ & $2006-2010$ & 2011-2014 \\
\hline $\begin{array}{l}\text { Tumor maligno de otros órganos } \\
\text { genitourinarios }\end{array}$ & 2,53 & 2,80 & 3,56 & 4,38 & 5,00 & 5,66 \\
\hline $\begin{array}{l}\text { Carcinoma in situ, tumores benignos y de } \\
\text { comportamiento incierto o desconocido }\end{array}$ & 1,29 & 3,88 & 6,09 & 1,36 & 3,89 & 6,57 \\
\hline $\begin{array}{l}\text { Tumor maligno de los órganos digestivos y } \\
\text { del peritoneo, excepto estómago y colon }\end{array}$ & 3,32 & 3,62 & 3,79 & 2,54 & 3,03 & 2,84 \\
\hline Demás del grupo & 3,86 & 3,84 & 4,13 & 3,07 & 3,42 & 3,68 \\
\hline \multicolumn{7}{|l|}{ Enfermedades transmisibles (Tasas $\times 100.000$ ) } \\
\hline Infecciones respiratorias agudas & 15,86 & 16,23 & 15,09 & 15,36 & 14,91 & 14,13 \\
\hline Enfermedad por el VIH/SIDA & 8,66 & 8,66 & 7,87 & 2,71 & 2,68 & 2,83 \\
\hline Tuberculosis, inclusive secuelas & 4,61 & 3,97 & 3,23 & 2,64 & 2,10 & 1,51 \\
\hline Septicemia & 3,41 & 2,75 & 2,86 & 3,16 & 2,63 & 2,79 \\
\hline Enfermedades infecciosas intestinales & 3,54 & 2,84 & 1,45 & 3,66 & 2,93 & 1,66 \\
\hline Otras enfermedades infecciosas y parasitarias & 1,61 & 1,55 & 1,36 & 1,36 & 1,46 & 1,15 \\
\hline Meningitis & 1,58 & 1,23 & 0,87 & 1,49 & 0,84 & 0,48 \\
\hline Demás del grupo & 1,61 & 0,92 & 0,73 & 1,12 & 0,64 & 0,52 \\
\hline \multicolumn{7}{|c|}{ Ciertas afecciones originadas en el período perinatal (Tasas $\times 1.000 \mathrm{NV})$} \\
\hline $\begin{array}{l}\text { Trastornos respiratorios específicos del } \\
\text { período perinatal }\end{array}$ & 4,25 & 3,67 & 2,87 & 3,72 & 2,80 & 2,23 \\
\hline $\begin{array}{l}\text { Otras afecciones originadas en el período } \\
\text { perinatal }\end{array}$ & 1,91 & 1,99 & 1,66 & 1,38 & 1,49 & 1,23 \\
\hline Sepsis bacteriana del recién nacido & 1,15 & 1,18 & 1,04 & 0,89 & 0,80 & 0,87 \\
\hline $\begin{array}{l}\text { Feto y recién nacido afectados por } \\
\text { complicaciones obstétricas y traumatismo del } \\
\text { nacimiento }\end{array}$ & 1,24 & 0,96 & 0,61 & 0,89 & 0,74 & 0,53 \\
\hline $\begin{array}{l}\text { Feto y recién nacido afectados por ciertas } \\
\text { afecciones maternas }\end{array}$ & 0,33 & 0,34 & 0,33 & 0,31 & 0,28 & 0,23 \\
\hline Demás del grupo & 0,45 & 0,23 & 0,49 & 0,37 & 0,18 & 0,34 \\
\hline
\end{tabular}

N/A: No aplica; NV: Nacidos vivos

\section{Enfermedades del sistema circulatorio}

En general, durante el periodo 2002-2014, la mortalidad por enfermedades del sistema circulatorio mostró una tendencia ascendente en Cauca y Nariño (véase figura 3a), siendo estadísticamente significativa en Nariño, tanto en la población total (de 89,6 a 110,0) como en hombres (de 87,6 a 104,5) y mujeres (de 91,6 a 115,6).

En este grupo predominaron, en ambos sexos, las enfermedades isquémicas del corazón (tendencia ascendente), las cerebrovasculares y las hipertensivas (véase tabla 4); estas dos últimas con una tendencia casi estable.

El análisis según sexo mostró que la tasa de mortalidad por enfermedades del sistema circulatorio fue ligeramente mayor en hombres en Valle del Cauca, mientras que en Chocó y Nariño fue mayor en mujeres (véase figura 3a). Las razones de mortalidad (hombre/ mujer) promedio fueron 1,11 en Valle del Cauca; 1,03 en Cauca; 0,91 en Nariño, y 0,89 en Chocó.

\section{Todas las demás causas}

La tendencia 2002-2014 de la mortalidad en el grupo de todas las demás causas fue ascendente en Nariño y Valle del Cauca (véase figura 3b). Las tasas totales mostraron una tendencia ascendente y estadísticamente significativa en Nariño (de 76,3 a 104,9) y Valle del Cauca (de 116,3 a 129,0), al igual que en hombres (de 72,7 a 101,9 ) y mujeres (de 79,9 a 108,0) de Nariño.

Dentro de este grupo se destaca la mortalidad por enfermedades crónicas de las vías respiratorias inferiores (leve tendencia ascendente), diabetes mellitus (tendencia descendente) y resto de enfermedades del sistema digestivo (tendencia ascendente) (véase tabla 4).

En este grupo, las tasas de mortalidad entre hombres y mujeres mostraron un patrón similar en los cuatro departamentos, con razones de mortalidad (hombre/ mujer) promedio alrededor de 1 .

\section{Causas externas}

La mortalidad por causas externas mostró una clara tendencia al descenso en los cuatro departamentos de 2002 a 2014, especialmente en hombres (véase figura $3 c)$. Este descenso fue estadísticamente significativo en Valle del Cauca, tanto en la población general (de 147,5 a 89,1 ) como en hombres (de 272,4 a 163,5 ) y mujeres 
(de 29,0 a 19,1), como también en las mujeres del Cauca (de 26,6 a 19,6).

Dentro del grupo de causas externas predominaron los homicidios, accidentes de transporte terrestre y suicidios (véase tabla 4). Durante los tres periodos se observó un claro descenso en las tasas de mortalidad por homicidios en hombres, aunque también en mujeres. Las tasas de mortalidad por accidentes de transporte terrestre también se redujeron en ambos sexos, mientras que con los suicidios, el descenso fue más evidente en el sexo femenino.

En todos los departamentos, las tasas de mortalidad por causas externas fueron mayores en los hombres que en las mujeres, con razones promedio de tasas (hombre/ mujer) de 5 en Nariño, 6 en Cauca, 7 en Chocó y 9 en Valle del Cauca (véase figura 3c).

\section{Neoplasias}

Se observó una tendencia ascendente en la mortalidad por neoplasias en los cuatro departamentos. La tendencia ascendente fue estadísticamente significativa en Nariño, tanto en la población general (de 50,4 a 69,2) como en hombres (de 45,1 a 67,3) y mujeres (de 55,8 a 71,1).

Este grupo de mortalidad incluye varios tipos de tumores, destacándose en los hombres el tumor maligno del estómago (tendencia descendente), próstata (tendencia ascendente), otros tumores malignos (tendencia estable) y tumor maligno de la tráquea, los bronquios y el pulmón (tendencia descendente) (véase tabla 4). En las mujeres, también se destacó el tumor maligno del estómago (tendencia descendente), otros tumores malignos (tendencia estable), del útero (tendencia descendente) y de la mama (tendencia ascendente).

Las tasas específicas de mortalidad por neoplasias entre hombres y mujeres fueron muy similares en los cuatro departamentos, con razones de mortalidad (hombre/mujer) promedio alrededor de 1.

\section{Enfermedades transmisibles}

Todas las tasas de mortalidad por enfermedades transmisibles mostraron una tendencia descendente, que fue estadísticamente importante en Cauca, Nariño y Valle del Cauca. En la población total, las tasas descendieron, durante el periodo de estudio, en Cauca (de 34,1 a 16,7), Nariño (de 23,5 a 16,7) y Valle del Cauca (de 37,9 a $34,8)$. Según el sexo, las tasas de mortalidad también descendieron, en Cauca (hombres de 38,2 a 18,2; mujeres de 29,9 a 15,1), Nariño (hombres de 23,9 a 15,7; mujeres de 23,0 a 17,6) y Valle del Cauca (hombres de 45,9 a 40,1; mujeres de 30,4 a 29,7).

En este grupo, la principal causa de muerte en hombres y mujeres fueron las infecciones respiratorias agudas (tendencia descendente) (véase tabla 4). En los hombres, también se destacó la mortalidad por virus de inmunodeficiencia humana ( $\mathrm{VIH})$ / síndrome de inmunodeficiencia adquirida (SIDA) (tendencia estable) y por tuberculosis (tendencia descendente). En las mujeres, se destacaron las tasas de mortalidad por enfermedades infecciosas intestinales (tendencia descendente), mientras la mortalidad por VIH/SIDA tuvo un leve ascenso.

En general, las tasas de mortalidad por enfermedades transmisibles fueron mayores en hombres que en mujeres, principalmente en Valle del Cauca. Las razones de mortalidad (hombre/mujer) promedio fueron de 1,12 en Nariño; 1,21 en Cauca; 1,24 en Chocó, y 1,44 en Valle del Cauca.

\section{Ciertas afecciones originadas en el periodo perinatal}

La mortalidad del grupo "perinatal" incluye las defunciones por causas originadas en el periodo perinatal (hasta el $7 .^{\circ}$ día de vida), aunque la muerte ocurra después.

En general, las tendencias de mortalidad de este grupo fueron descendentes durante el periodo 2002-2014 en todos los departamentos (véase figura 3f), aunque el descenso no fue estadísticamente significativo. Las tasas totales de mortalidad $(\times 1.000 \mathrm{NV})$ descendieron en Cauca (de 11,8 a 7,9), Chocó (de 18,8 a 12,1), Nariño (de 11,4 a 5,9) y Valle del Cauca (de 7,5 a 4,7).

Dentro de las causas de muerte perinatales se destacó la mortalidad por trastornos respiratorios específicos del período perinatal (tendencia descendente) (véase tabla 4). También se encontraron tendencias descendentes por otras afecciones originadas en el período perinatal y por sepsis bacteriana del recién nacido.

En este grupo se encontró mayor mortalidad entre los hombres, con razones de mortalidad (hombre / mujer) promedio de 1,16 en Chocó, 1,27 en Cauca, 1,28 en Nariño y 1,35 en Valle del Cauca.

\section{Discusión}

Se hizo un análisis de datos secundarios para describir la magnitud y la tendencia de la mortalidad general y específica, durante el periodo 2002-2014, en los departamentos de la región del Pacífico colombiano.

Se encontró una ligera tendencia descendente en las tasas estandarizadas de mortalidad en la región. Este comportamiento es coherente con varios estudios y reportes para Colombia, donde, en general, se ha observado un descenso de la mortalidad y un aumento de la esperanza de vida en las últimas décadas [26]. En parte, esta tendencia favorable puede entenderse como un reflejo de los avances en el campo de la medicina y la salud pública, mejores condiciones de vida y acceso a atención en salud $[1,7,26]$. 
El análisis de la mortalidad según el sexo mostró mayor mortalidad masculina en los cuatro departamentos, similar a lo hallado en Colombia durante el periodo 2005-2014 (56,9 \%). La sobremortalidad masculina, principalmente entre los 15 a 44 años, puede atribuirse al grupo de causas externas que presentó razones de mortalidad (hombre / mujer) entre 5 y 9. Este es un fenómeno uniforme en todos los países de Latinoamérica, según un informe de la ops [27] y algunos estudios nacionales [28] o internacionales [29], y ha sido explicado previamente por el papel de la "masculinidad" en la sociedad contemporánea, que ha llevado al hombre a la adopción de conductas más agresivas y de mayor riesgo para su vida, como el uso de alcohol y sicoactivos, conducir bajo los efectos de estas sustancias o la participación en riñas o conflictos armados [30].

Los resultados de mortalidad por causas externas encontrados confirman que, en Colombia, incluyendo los departamentos de la región del Pacífico, predomina la mortalidad por homicidios, accidentes de tránsito y suicidios [6]. Las causas externas fueron la tercera causa de muerte en los departamentos de la región del Pacífico y la cuarta en Colombia entre 2005 y 2014 [7]. Esta región tiene un aporte importante a la mortalidad nacional por causas externas, que puede ser explicado en términos del conflicto armado, donde históricamente los departamentos del Pacífico han sido escenario de la guerra interna en Colombia [31].

El comportamiento decreciente en la mortalidad por causas externas es concordante con el nivel nacional [7] y lo reportado en algunos estudios descriptivos $[13,26]$ y comparativos [10]. Puede ser explicado parcialmente por la disminución de homicidios reportado en Colombia desde el 2002, impulsado por el establecimiento del "Plan Colombia", la desmovilización de grupos armados, la implementación de estrategias/políticas para la restricción de consumo de alcohol y porte de armas [13], y planes de seguridad vial encaminados a reducir la accidentalidad [12]. Todos estos acontecimientos también ayudan a explicar la tendencia descendente de la mortalidad masculina y la general.

Las principales causas de muerte encontradas en este estudio (enfermedades del sistema circulatorio, todas las demás causas, causas externas y neoplasias) coinciden con el estudio de cambios en las principales causas de mortalidad en Colombia de 1990 a 2012 de Acosta y Romero, efectuado en 2014 [26]. Sin embargo, el peso relativo de cada grupo difiere por departamento, con diferencias en la mortalidad general y específica, aun dentro de una misma región. La transformación del perfil de mortalidad hacia las enfermedades crónicas no transmisibles obedece al proceso de envejecimiento de la población y al aumento de la prevalencia de las enfermedades crónicas, debido seguramente a los avances tecnológicos y científicos para abordar el cáncer y las enfermedades circulatorias.

La principal causa de muerte en los departamentos en estudio correspondió al grupo de enfermedades del sistema circulatorio, similar a Colombia durante el periodo 2005 a 2014 [7]. Las principales causas de muerte coincidieron con lo reportado por la oms en 2015 a nivel mundial, donde predominó la mortalidad por enfermedades isquémicas del corazón y cerebrovasculares [5]. La tendencia ascendente en la mortalidad por enfermedades del sistema circulatorio fue similar a la reportada para Colombia entre 1990 y 2012 [26].

En el grupo de todas las demás causas predominó la mortalidad por enfermedades crónicas de las vías respiratorias inferiores y diabetes mellitus, hallazgos consistentes con resultados para Colombia [7]. Llamó la atención la tendencia ascendente en las tasas de mortalidad por neoplasias en los cuatro departamentos, tanto en hombres como en mujeres. El cáncer de estómago también ha sido reportado previamente como el principal causante de muerte en Colombia y, similar a nuestro estudio, con una tendencia al descenso $[32,33]$. Las tendencias encontradas en otros tipos de cáncer, como próstata en hombres (ascendente) y útero (descendente) y mama (ascendente) en mujeres, igualmente son consistentes con otros estudios para Colombia [32,33].

Las tendencias ascendentes en los grupos de enfermedades del sistema circulatorio, demás causas y neoplasias son coherentes con el proceso de transición demográfica y epidemiológica en las Américas, en cuanto al aumento ponderado de las enfermedades crónicas y degenerativas. Su tendencia ascendente es explicada parcialmente por cambios en los factores de riesgo de las enfermedades no transmisibles, y puede estar relacionado, entre otros, con el envejecimiento poblacional, malos hábitos alimenticios, sedentarismo y el aumento de la obesidad en estos departamentos [34, p. 59], determinantes altamente relacionados con las enfermedades no transmisibles.

Los cuatro departamentos mostraron una tendencia descendente en la mortalidad por enfermedades transmisibles, comportamiento coherente con el nivel nacional [7], lo que es explicado parcialmente por mejores condiciones de vida. La mayor mortalidad en hombres puede ser explicada por el aporte masculino a la carga de enfermedad/muerte por VIH/SIDA, tuberculosis y enfermedad respiratoria aguda, que históricamente ha sido mayor en hombres que en mujeres [35].

En el grupo perinatal, los trastornos respiratorios específicos del período perinatal fueron la principal causa de muerte y con una tendencia descendente similar a lo reportado en Colombia para el periodo 2005 a 2014 [7]. Este descenso podría ser explicado, entre otras razones, 
por mejoras en las condiciones de vida de la población y la nutrición materna, y por avances en términos de la salud materna (acceso, oportunidad y calidad de los servicios de atención a cuidados prenatales, atención del parto y del recién nacido) [36]. Sin embargo, los niveles de subregistro de la mortalidad infantil y perinatal podrían dar una imagen errada de este descenso.

El perfil epidemiológico de Chocó difirió de los otros departamentos, al presentar las mayores tasas de mortalidad del grupo perinatal, como también en niños menores de 1 año, de 1 a 4 y de 5 a 9 años. Este perfil de mortalidad es coherente, al ser el departamento con la estructura poblacional más joven y con la mayor carga de mortalidad por enfermedades transmisibles, en contraste con Valle del Cauca, donde tuvieron más peso las causas de muerte relacionadas con las enfermedades no transmisibles. Estos resultados son también coherentes con el nivel de desarrollo económico de estos departamentos, donde Valle del Cauca es el tercer departamento más industrializado de Colombia y Chocó es uno de los más marginados económica y socialmente, con problemas de infraestructura de servicios especializados para la atención materno perinatal y con las condiciones de vida más precarias, como lo muestran los indicadores de la tabla 1. Este nivel de desarrollo asimismo podría explicar el hecho de que las mayores tasas de mortalidad (general y específica) pertenecieron al Valle del Cauca, por contar con un mejor sistema de registro de información.

Una limitación importante de este estudio está relacionada con el uso de información proveniente de fuentes secundarias (datos de mortalidad, tamaños de población y nacimientos) en cuanto a calidad y cobertura. Para los datos de mortalidad, la información se consideró de alta calidad de acuerdo con los resultados del software ANACONDA $^{\circledR}$, además que solo fueron excluidos el $0,66 \%$ de los registros, por tener información demográfica faltante (sexo, edad o municipio), y solo el 1,8\% de las defunciones fueron clasificadas con causa de mortalidad "mal definida". En cuanto a cobertura, la información oficial disponible del último Censo en Colombia (Censo DANE 2005) [37] exploró las defunciones con certificado de defunción, encontrando una cobertura del 83,8 \% en Cauca, 84,3 \% en Chocó, 90,2 \% en Nariño y $94,8 \%$ en Valle del Cauca. Dado que la mortalidad presenta un comportamiento relativamente constante, llamó la atención el descenso repentino que se observó en la mortalidad (general y específica) en Nariño para el año 2005, lo que hace pensar en un subregistro de la información. Aunque se validó la consistencia de la información, el subregistro parece ocultar la verdadera magnitud de la mortalidad.

\section{Conclusiones}

El perfil de mortalidad en la región del Pacífico de Colombia mostró un descenso en la mortalidad general en los departamentos de Cauca, Nariño y Valle del Cauca. Según sexo, la mortalidad masculina fue mayor que la femenina, pero con una tendencia descendente. Por edad, el riesgo diferencial entre hombres y mujeres comienza a partir del grupo de los 15 a 19 años, atribuido al grupo de causas externas.

Durante todo el periodo, la principal causa de muerte fueron las enfermedades del sistema circulatorio que, al igual que el grupo de todas las demás causas y neoplasias, aumentaron su participación como causa de muerte. Por el contrario, el grupo de causas externas presentó un marcado descenso, así como el grupo de enfermedades transmisibles y afecciones originadas en el periodo perinatal.

El perfil de mortalidad de Valle del Cauca se asemejó más al de regiones desarrolladas, mientras que, en Chocó, el peso importante de la mortalidad en niños refleja su rezago económico y social en la región. Las tendencias de mortalidad fueron propias de cada región, mostrando que existen desigualdades en la mortalidad.

Los problemas de cobertura y certificación de los hechos vitales en los departamentos de Chocó, Nariño y Cauca requieren fortalecimiento de la gestión del subsistema de estadísticas vitales, para mejorar su cobertura y calidad. Se requieren metodologías para aproximarse a la comprensión de las dinámicas de la mortalidad de unidades poblacionales pequeñas y con datos limitados.

\section{Conflicto de interés}

Los autores declaramos no tener conflicto de intereses.

\section{Financiación}

Este estudio fue financiado por las universidades: Pontificia Universidad Javeriana, Cali, Colombia; Universidad Externado de Colombia, Bogotá, Colombia; y Universidad de São Paulo, Ribeirão Preto, Brasil.

\section{Declaración de responsabilidad}

Se declara que los puntos de vista expresados en este artículo son responsabilidad de los autores y no de las universidades donde trabajan. 


\section{Declaración de autoría}

Mauricio Pérez-Flórez concibió la idea y diseño del estudio, realizó la interpretación de los datos, elaboró la primera versión del manuscrito, aprobó la versión final que se publica y se hace responsable por todos los aspectos del estudio.

Jorge Alberto Achcar concibió la idea y diseño del estudio, revisó críticamente el manuscrito, aprobó la versión final que se publica y se hace responsable por todos los aspectos del estudio.

Isabel Cristina Ruiz Buitrago consiguió información importante para verificar la calidad de la información, revisó críticamente el manuscrito, aprobó la versión final que se publica y se hace responsable por todos los aspectos del estudio.

\section{Referencias}

1. Centro Latinoamericano y Caribeño de Demografía (CELADE), Comisión Económica para América Latina y el Caribe (CEPAL), Observartorio demográfico. Mortalidad. [internet]; 2007. [citado 2018 oct. 10]. Disponible en: https://repositorio.cepal.org/ bitstream/handle/11362/7121/1/S0701080_mu.pdf

2. McKeown RE. The Epidemiologic Transition: Changing Patterns of Mortality and Population Dynamics. Am. J. Lifestyle Med. 2009;3(1 Suppl.):19S-26S

3. Organización Panamericana de la Salud. Transición demográfica en las Américas. Boletín Epidemiológico [internet] 1994;15(1):45-8. [citado 2017 ago. 28]. Disponible en: http:// iris.paho.org/xmlui/bitstream/handle/123456789/32187/18847. pdf? sequence $=1 \&$ isAllowed $=y$

4. Frenk J, Frejka T, Bobadilla JL, et al. La transición epidemiológica en América Latina. Bol. Oficina Sanit. Panam. [internet]. 1991 [citado 2017 ago. 17]; 6(111):485-96. Disponible en: http:// iris.paho.org/xmlui/bitstream/handle/123456789/16560/ v111n6p485.pdf? sequence $=1$ \&isAllowed $=\mathrm{y}$

5. Organización Mundial de la Salud (oms). Las 10 principales causas de defunción [internet]. 2018 [citado 2017 sep. 11]. Disponible en: http://www.who.int/mediacentre/factsheets/ fs $310 /$ es/

6. Di Cesare M. El perfil epidemiológico de América Latina y el Caribe: desafíos, límites y acciones. Comisión Económica para América Latina y el Caribe (cepal) [internet]; 2011 [citado 2017 sep. 4]. Disponible en: https://repositorio.cepal.org/bitstream/ handle/11362/3852/1/S2011938.pdf

7. Ministerio de Salud y Protección Social, Dirección de Epidemiología y Demografía. Grupo asis. Análisis de Situación de Salud (asis) Colombia, 2016 [internet]; 2016 [citado 2018 abr. 13]. Disponible en: https://www.minsalud.gov.co/sites/rid/Lists/ BibliotecaDigital/RIDE/VS/ED/PSP/asis-colombia-2016.pdf

8. Evans R, Pérez J, Bonilla R. Análisis de la mortalidad por enfermedades cerebrovasculares en Costa Rica entre los años 1920-2009. Arch. Cardiol. México [internet]. 2016 [citado 2017 sep. 3]; 86(4):358-66. Disponible en: http://linkinghub.elsevier. com/retrieve/pii/S1405994016300404. doi: http://dx.doi. org/10.1016/j.acmx.2016.05.009
9. Hernández H, Macías G. Análisis de la tendencia temporal de la mortalidad por diabetes mellitus en Argentina, 1990-2013. Rev. Panam. Salud Pública. 2017;41(5):1-7.

10. Cardona Arango D, Escané G, Fantín MA, et al. Mortalidad por causas externas: un problema de salud pública. Argentina, Chile y Colombia. 2000-2008. Población y Salud en Mesoamérica. 2013;10(2):1-13

11. Cardona D, Peláez E, Aidar T, et al. Mortalidad por causas externas en tres ciudades latinoamericanas: Córdoba (Argentina), Campinas (Brasil) y Medellín (Colombia), 1980-2005. Rev. Bras. Estud. Popul. 2008;25(2):335-52. doi: http://dx.doi.org/10.1590/ S0102-30982008000200009

12. Cardona AMS, Arango DC, Fernández DYB, et al. Mortality in traffic accidents with older adults in Colombia. Rev. Saúde Pública [internet]. 2017 [citado 2017 sep. 1]; 51:1-21. Disponible en: http://www.scielo.br/scielo.php?script=sci arttext\&pid=S0034$89102017000100216 \& \operatorname{lng}=$ en\&nrm=iso\&tlng=en\%5Cnhttp:// www.ncbi.nlm.nih.gov/pubmed/28355347\%5Cnhttp://www. pubmedcentral.nih.gov/articlerender.fcgi?artid=PMC5344075. doi: http://dx.doi.org/10.1590/s1518-8787.2017051006405

13. Chaparro-Narváez P, Cotes-Cantillo K, León-Quevedo W, et al. Mortalidad por homicidios en Colombia, 1998-2012. Biomédica [internet]. 2016 [citado 2017 sep. 1]; 36(4):572-82. Disponible en: http://www.revistabiomedica.org/index.php/biomedica/ article/view/2811. doi: https://doi.org/10.7705/biomedica. v36i4.2811

14. Bravo LE, García LS, Carrascal E, et al. Burden of breast cancer in Cali, Colombia: 1962-2012. Salud Publica Mex. 2014;56(5):44856. doi: https://doi.org/10.21149/spm.v56i5.7370

15. Maria Virgínio de Sousa A, Carla Alves Teixeira C, da Silva Medeiros S, et al. Mortalidade por câncer do colo do útero no estado do Rio Grande do Norte, no período de 1996 a 2010: tendência temporal e projeções até 2030. Epidemiol e Serviços Saúde [internet]. 2016 [citado 2017 sep. 3]; 25(2):112. Disponible en: http://www.scielo.br/pdf/ress/v25n2/22379622-ress-25-02-00311.pdf doi: https://doi.org/10.5123/S167949742016000200010

16. Cardona D, Agudelo HB. Tendencias de mortalidad en población adulta, Medellín 1994-2003. Biomédica [internet]. 2007;27(3):352-63. [citado 2017 Jul. 6]. Disponible en: https:// revistabiomedica.org/index.php/biomedica/article/view/198. doi: https://doi.org/10.7705/biomedica.v27i3.198

17. Departamento Administrativo Nacional de Estadística (DANE) Colombia. Proyecciones de población municipales por área 2005-2020 [internet]. 2011 [citado 2017 sep. 11]. Disponible en: https://goo.gl/5Ghk8n

18. Sociedad Geográfica de Colombia. Geografía de Colombia, Departamentos de Colombia, Fichas temáticas [internet]; 2011 [citado 2017 nov. 11]. Disponible en: http://www.sogeocol.edu. co/dptosyciud.htm

19. Departamento Nacional de Planeación. Indicadores sociales departamentales. Boletines Sistema de Indicadores Sociodemográficos. [internet]; 2005 [citado 2017 nov. 11]. Disponible en: https://colaboracion.dnp.gov.co/CDT/Desarrollo Social/boletin37.pdf

20. Departamento Administrativo Nacional de Estadística (DANE). Estadísticas vitales [internet]; s. f. [citado 2017 apr. 11]. Disponible en: http://microdatos.dane.gov.co/index.php/catalog/ MICRODATOS/about_collection/22/5

21. Pan American Health Organization (paho). New paho List 6/67 for Tabulation of icd-10 Mortality Data. Epidemiol Bull 
[internet]; 1999. [citado 2017 abr. 30]; 20(3):4-9. Disponible en: http://iris.paho.org/xmlui/handle/123456789/31900

22. Naghavi M, Wang H, Lozano R, et al. Global, regional, and national age-sex specific all-cause and cause-specific mortality for 240 causes of death, 1990-2013: A systematic analysis for the Global Burden of Disease Study 2013. Lancet [internet]. 2015 [citado 2017 nov. 17]; 385(9963):117-71. Disponible en: https://www.thelancet.com/journals/lancet/article/PIIS01406736(14)61682-2/fulltext. doi: http://dx.doi.org/10.1016/S01406736(14)61682-2

23. Phillips DE, Lozano R, Naghavi M, et al. A composite metric for assessing data on mortality and causes of death: The vital statistics performance index. Popul. Health Metr. [internet]. 2014 [citado 2017 nov. 17] ;12(1):14. Disponible en: http://pophealthmetrics. biomedcentral.com/articles/10.1186/1478-7954-12-14

24. Spiegelhalter DJ, Thomas A, Best NG, et al. WinBugs: user manual, version 1.4. Cambridge, u.k.: mrc Biostatistics Unit, editor. 2003.

25. Paulino C., Turkman M, Murteira B. Estatística bayesiana Lisboa: Fundação Calouste Gulbenkian; 2003.

26. Acosta K, Romero J. Cambios recientes en las principales causas de mortalidad en Colombia. Documentos de Trabajo Sobre Economía Regional. Cartagena: Banco de la República Sucursal Cartagena; 2014.

27. Organización Panamericana de la Salud. Salud en las Americas 2007 Volumen I - Regional. N. 622 [internet]; 2007 [citado 2018 may. 16]. Disponible en: http://www.paho.org/cor/index. php?option $=$ com_docman\&task $=$ doc_view\&gid $=256 \&$ Itemid $=$

28. Caicedo-Velásquez B, Álvarez-Castaño LS, Marí-Dell’Olmo $\mathrm{M}$, et al. Evolución de las inequidades en mortalidad por causas externas entre los municipios de Antioquia (Colombia). Gac. Sanit. 2016;30(4):279-86. doi: http://dx.doi.org/10.1016/j. gaceta.2016.02.010

29. Moura EC de, Gomes R, Falcão MTC, et al. Gender inequalities in external cause mortality in Brazil, 2010. Ciênc. Saúde Colet [internet]. 2015 [citado 2017 sep. 15]; 20(3):779-88 Disponible en: http://www.scielo.br/scielo.php?script=sci arttext\&pid=S1413-81232015000300779\&lng=en\&tlng=en doi: http://dx.doi.org/10.1590/1413-81232015203.11172014
30. Sorenson SB. Gender disparities in injury mortality: Consistent, persistent, and larger than you'd think. Am J Public Health. 2011;101(Suppl. 1):S353-S8. doi: http://dx.doi.org/10.2105/ AJPH.2010.300029

31. Chará Ordónez WD. Las víctimas del conflicto armado interno en Colombia, 1985-2015. Anuari del Conflicte Social 2015 [internet]. 2015 [citado 2017 sep. 18]; 47-80. Disponible en: http://revistes.ub.edu/index.php/ACS/article/ viewFile/16002/19048http://revistes.ub.edu/index.php/ACS/ article/viewFile/16002/19048

32. Piñeros $\mathrm{M}$, Gamboa $\mathrm{O}$, Hernández-Suárez $\mathrm{G}$, et al. Patterns and trends in cancer mortality in Colombia 1984-2008. Cancer Epidemiol. 2013;37(3):233-9. doi: http://dx.doi.org/10.1016/j. canep.2013.02.003

33. Pardo C, de Vries E, Buitrago L, et al. Atlas de mortalidad por cáncer en Colombia (4.a ed. Bogotá: Instituto Nacional de Cancerología, Instituto Geográfico Agustín Codazzi; 2017.

34. Colombia, Ministerio de Salud y Protección Social, Dirección de Epidemiología y Demografía, Grupo asis. Análisis de situación de salud según regiones Colombia; 2013.

35. Gonzalez MA, Martin L, Munoz S, Jacobson JO. Patterns, trends and sex differences in hiv/aids reported mortality in Latin American countries: 1996-2007. bmc Public Health [internet]. 2011 [citado 2017 sep. 18]; 11(1):605. Disponible en: http:// www.pubmedcentral.nih.gov/articlerender.fcgi? $\operatorname{artid}=31733$ 48\&tool=pmcentrez\&rendertype $=$ abstract $\quad$ doi: http://dx.doi. org/10.1186/1471-2458-11-605

36. Colombia. Ministerio de Salud y Protección Social, y Profamilia. (2017). Publicación Encuesta Nacional de Demografía y Salud. ends; 2015. [citado 2018 may. 1]. Disponible en: https://www. minsalud.gov.co/sites/rid/Lists/BibliotecaDigital/RIDE/VS/ED/ GCFI/libro-ends-2015.zip

37. Departamento Nacional de Estadística (DANE). Censo General 2005 [internet]; 2005 [citado 2018 oct. 10]. Disponible en: https://www.dane.gov.co/index.php/estadisticas-por-tema/ demografia-y-poblacion/censo-general-2005-1 